\title{
Loyale Konspiratoren
}

\author{
Die Verschwörung bulgarischer Offiziere 1886/87
}

von

Deniza Petrova

\begin{abstract}
Das Wirken des konspirativen militärischen Netzwerks in Bulgarien 1886/1887 gestaltete sich in der Spannung zwischen persönlicher Identität, nationalen Prämissen und politischer Loyalität. Gelegentlich harmonierten diese Faktoren miteinander, in dem beschriebenen Fallbeispiel divergierten sie aber in einem so bohen Maße, das dadurch militärischer Korpsgeist, Berufsethos und Loyalität zum eigenen Staat und zum Monarchen als oberstem Befehlshaber ausgehebelt wurden. Die gespaltene Loyalität des bulgarischen Militärs bildete den Ausgangspunkt für die Verschwörung einer Gruppe von Offizieren gegen den bulgarischen Fürsten und deutschen Prinzen Alexander von Battenberg. Zum einen spielte die Vergangenheit der Verschwörer als Mitglieder eines geheimen Netzwerkes, in dem sie erste Erfahrungen im Untergrundkampf und in der Geheimbaltung sammeln konnten, eine wesentliche Rolle. Zum anderen bauten sie eine militärische Karriere auf und wurden von Rebellen gegen staatliche Gewalt zu legitimen Schützern des Staates, um dann sich wiederum gegen den Staat aufzulehnen. Einerseits waren sie - die früheren Kämpfer gegen das Osmanische Reich - bulgarische Patrioten. Andererseits füblten sie sich ibren russischen Ausbildern und Vorgesetzten verbunden und wurden zu Instrumenten russischer Machtpolitik in Bulgarien. Ibre Loyalität galt Russland und nicht dem bulgarischen Fürsten; diesen saben sie als ersetzbare Marionette der europäischen Großmächte, welche zur Gefahr für die Allianz mit Russland und hiermit für das Wobl Bulgariens geworden war. In diesem Sinne agierten sie 1886/1887 als loyale Konspiratoren - mit ibrer Identität im geheimen Untergrund verwurzelt, ibrem Vaterland und einem fremden Staat zugleich loyal. Der Putsch und seine Hintergründe verdeutlichen die Komplexität und die Sprengkraft geheimer Netzwerke innerhalb des Militärs. Die trennende Wirkung solcher Gruppierungen entfaltete sich im gespaltenen Identitätskonstrukt, welches das bulgarische Heer in den Jahren unmittelbar nach seiner Entstehung charakterisierte.
\end{abstract}

Die Entstehung der Nationalstaaten auf dem Balkan im 19. und 20. Jahrhundert ging mit zahlreichen bewaffneten Konflikten, Kriegen und inneren Krisen einher ${ }^{1}$. Die berüchtigte Instabilität, die das Klischee »Pulverfass Europas « prägte, wurde hauptsächlich durch zwei Faktoren bewirkt und beeinflusst: zum einen suchten die einzelnen neu gebildeten Staaten ihre territorialen Ansprüche und machtpolitische Position in der Region zu wahren und zu verfes-

Allgemein zur Nationalstaatenbildung auf dem Balkan vgl. Mark Mazower, The Balkans, London 2000; Steven W. Sowards, Moderne Geschichte des Balkans. Der Balkan im Zeitalter des Nationalismus, Seuzach 2004; Edgar Hösch, Geschichte des Balkans, 3. ergänzte Aufl., München 2011; Mark Biondich, The Balkans. Revolution, War and Political Violence since 1878, Oxford 2011. 
tigen, zum anderen gelangte der Balkan verstärkt in den Blick der europäischen Großmächte und wurde zur Arena für ihre Interessenspolitik²

Zum Garant der nationalen Souveränität und zur gesellschaftlichen Elite in Bulgarien entwickelte sich in dieser Zeit des Umbruchs immer mehr das Militär, welches sich noch in den Kinderschuhen befand ${ }^{3}$. Es betrachtete sich selbst als symbolischen Nachfolger der Heere und Heerführer des mittelalterlichen bulgarischen Reiches. Von dieser beanspruchten Tradition war allerdings infolge der knapp fünf Jahrhunderte währenden osmanischen Herrschaft kaum mehr übrig geblieben als einige alte Chroniken zur altbulgarischen Kriegsgeschichte. In der Realität weit wirksamer waren die Tradition der Heiduken, ihre Erfahrungen im Umgang mit der Waffe und ihre Ausformungen von »Ehrenkodizes«. Diese Freischärler agierten vom 15. bis weit ins 19. Jahrhundert hinein als Widerstandskämpfer gegen die osmanischen Landesherren.

Eine wichtige Rolle spielte schließlich die 1869 unter der Führung von Vasil Levski $^{4}$ entstandene Innere Revolutionäre Organisation (IRO) und das hierzu gehörende Bulgarische Revolutionäre Zentralkomitee $(B R Z K)^{5}$ mit Sitz in Bukarest. Die Mitglieder der IRO fühlten sich der Sache eines unabhängigen bulgarischen Nationalstaates verbunden, die sie über jegliche andere Verpflichtungen stellten. Ihr primäres Ziel war die Beschaffung von Waffen und Finanzmitteln sowie die militärische Ausbildung ihrer Mitglieder und eingeweihter Freiwilliger. 1870/71 umfasste die IRO etwa zweihundert geheime Revolutionskomitees im Landesinneren, die untereinander vernetzt waren. Dem BRZK in Bukarest kam dabei die Rolle der Steuerungszentrale zu. Führende Mitglieder der Komitees rekrutierten sich überwiegend aus dem Bürgertum und der Intelligenz, die ihre Ausbildung im Ausland, zumeist in Russland, erhalten hatten. Die IRO betrieb strenge Geheimhaltung, ihre Funktionäre bedienten sich in ihrer Korrespondenz einer Kodiersprache und verwendeten Pseudonyme und falsche Identitäten. Eine innere Geheimpolizei überprüfte die Integrität der Mitglieder, unternahm Maßnahmen gegen Verräter und sicherte die Organisation vor möglicher Infiltration durch die osmanische Polizei. Die Tätigkeit der IRO gipfelte in zwei Aufständen gegen die osmanische Herrschaft in Bulgarien, 1875 und 1876. Im darauf folgenden Russisch-Türkischen Krieg von 1877-78 kämpften unter russischem Kommando bulgarische Unabhängigkeitskämpfer und Exil-Revolutionäre, ehemalige Mitglieder der IRO, bulgarische Offiziere im russischen Dienst sowie Freiwillige.

2 Vgl. Rainer Schmidt, Die Balkankrise von 1875 bis 1878. Strategien der großen Mächte. In: Deutschland und Europa. Außenpolitische Grundlinien zwischen Reichsgründung und Erstem Weltkrieg. Festgabe für Harm-Hinrich Brandt zum siebzigsten Geburtstag, hrsg. von dems., Stuttgart 2004, S. 36-96.

3 Vgl. Vasil Popov, Cvetana Ivanova, Jonka Velkova, Bălgarskata zemska vojska 1878-1879, Sbornik ot dokumenti i materiali po săzdavaneto na bălgarskata vojska sled Osvoboditelnata ruskoturska vojna 1877-1878, Sofia 1959.

4 Über Rolle und Wirken von Vasil Levski siehe James Franklin Clarke, The Pen and the Sword. Studies in Bulgarian History, New York 1988 (= East European Monographs, 252), S. 151, 217; Richard J. Crampton, A Concise History of Bulgaria, Cambridge 1997, S. 78-81.

5 Vgl. Clarke, The Pen and the Sword (wie Anm. 4), S. 213, 219 
Die IRO, ihr Netzwerk und ihre Zielsetzung dienten als Vorbild für spätere Bewegungen ${ }^{6}$. Das Bulgarische Geheime Revolutionäre Zentralkomitee (BGRZK) setzte sich die Wiedervereinigung mit dem von Bulgarien nach dem Berliner Kongress ${ }^{7}$ abgetrennten Ost-Rumelien zum Ziel. In Mazedonien wirkte die Innere Mazedonische Revolutionäre Organisation (IMRO) von 1893 bis in die vierziger Jahre des 20. Jahrhunderts; in der Süd-Dobrudscha war nach dem Ersten Weltkrieg bis 1940 die Innere Dobrudschaner Revolutionäre Organisation (IDRO) tätig, welche die Wiederangliederung der Region an Bulgarien anstrebte ${ }^{8}$. All diese geheimen Netzwerke hatten gemeinsam, dass sie in engem Kontakt zum bulgarischen Militär standen bzw. bulgarische Militärs zu ihren Mitgliedern zählten oder dass ihre Mitglieder die militärische Laufbahn einschlugen?.

Abgesehen von dieser engen Verflechtung mit den bulgarischen Untergrundbewegungen prägte das bulgarische Militär seit seinen Anfängen eine enge Verbindung zu Russland. Die Verbände, die später den Kern des bulgarischen Heers bildeten, formierten sich während des Russisch-Türkischen Krieges $1877 / 78$, in dem sie auch Kampferfahrung sammeln konnten. Bereits vor dem Beginn der Kriegshandlungen wurden in Russland Einheiten aus bulgarischen Freiwilligen gebildet. Sie zogen als Teil der russischen Armee in den Krieg, geführt von russischen Offizieren. Im Herbst 1878, knapp zehn Monate nach dem Frieden von San Stefano, der den Krieg beendete und den bulgarischen Nationalstaat ins Leben rief, wurde die erste Offiziersschule Bulgariens gegründet. Im Jahr darauf hatten die ersten Kadetten ihre Ausbildung absolviert. Die Leitung und das Lehrpersonal der Schule setzten sich aus russischen Offizieren zusammen. Auch nach der Gründung eines eigenen bulgarischen Staates behielten die Russen ihre Kommandoposten im jungen bulgarischen Heer bei. Von 1879 bis 1885 waren die Kriegsminister Bulgariens russische Genera$1 \mathrm{e}^{10}$. Als »Befreier « und Kriegskameraden hatten sie einen enormen Einfluss und genossen eine nahezu sakrale Verehrung unter ihren Untergebenen. Allerdings waren die russisch besetzten Kommandostrukturen für bulgarische Offiziersanwärter wenig durchlässig und wurden so zur Karrierebremse für bulgarische Militärs ${ }^{11}$.

Der Berliner Kongress im Sommer 1878 zerteilte den neu geschaffenen, immer noch von russischen Truppen besetzten bulgarischen Staat. Es wurde ein unter osmanischer Souveränität stehendes tributpflichtiges Fürstentum eingerichtet und eine autonome Provinz Ost-Rumelien innerhalb des Osmanischen Reiches etabliert. Mazedonien wurde wieder dem Osmanischen Reich

6 Vgl. ebd., S. 195.

$7 \mathrm{Zu}$ den Folgen aus den Beschlüssen des Berliner Kongresses für Bulgarien siehe Richard J. Crampton, Bulgaria, Oxford 2007, S. 116-123, 150-152, 190-220.

8 Vgl. Ljubomir Zlatev, Vătrešnata dobrudžanska revoljucionna organizacija (VDRO) 1923-1940, Russe 2009.

9 Vgl. Svetlozar Eldărov, Tajnite oficerski bratstva v osvoboditelnite borbi na Makedonija i Odrinsko 1897-1912, Sofia 2002.

10 Vgl. Popov, Bălgarskata (wie Anm. 3), S. 60.

11 Vgl. Arthur von Huhn, Aus bulgarischer Sturmzeit. Eine authentische Darstellung des Handstreichs von Sofia und seiner Folgen, Leipzig 1886, S. 8 f. 
angegliedert. Erster Fürst von Bulgarien wurde der Neffe des russischen Zaren Alexander II., der deutsche Prinz Alexander von Battenberg - ein Konsenskandidat für den Thron aus der Sicht der europäischen Großmächte. Bald wandelte er sich trotz russischer Minister und Militärberater von einer bequemen Marionette zur Symbolfigur einer auf Selbstständigkeit ausgerichteten bulgarischen Nationalpolitik. Dies ließ das Verhältnis des jungen Staates zu Russland deutlich abkühlen. Die Krise vertiefte sich nach dem Tod Alexanders II. Sein Nachfolger Alexander III. betrieb gegenüber dem bulgarischen Fürstentum eine klare Dominanzpolitik, die zwar das Gleichgewicht der europäischen machtpolitischen Konstellationen in Europa zu wahren versuchte, Bulgarien aber weiterhin als wichtigen Baustein für zukünftige russische Expansion in Richtung Konstantinopel und Meerengen betrachtete ${ }^{12}$. »Nun sollen die Slawen Russland einen Dienst erweisen, und nicht Russland ihnen ${ }^{13}$ «, schrieb Alexander III. seinem Generalstabs-Chef Nikolai Obrutschew.

In den ersten Septembertagen 1885 setzte sich Alexander von Battenberg an die Spitze einer Bewegung, welche die Vereinigung des Fürstentums Bulgarien mit Ost-Rumelien vollzog und sich hiermit gegen die Beschlüsse des Berliner Kongresses wandte und den von den europäischen Großmächten geregelten Status-quo verletzte. Bulgarien war es damit gelungen, die aufgezwungene Zerteilung zu überwinden, es sah sich nun aber weitgehend mit außenpolitischer Isolation und vor allem mit dem Widerstand Russlands konfrontiert. Die damals populärste russische Zeitung Moskovskie vedomosti, redigiert vom Verfechter des Panslawismus ${ }^{14}$ Michail Katkow, schrieb im März 1886: »Der einzige Ausweg aus der Krise ist eine Okkupation Bulgariens. In Bulgarien brauchen wir keinen diplomatischen Vertreter bei der illegitimen Regierung, sondern einen Kommissar mit diktatorischer Vollmacht für ein oder zwei Jahre ${ }^{15} \cdot \ll$

In dem darauf folgenden Serbisch-Bulgarischen Krieg vom 02.-16.11. jul. $1885^{16}$ gelang den bulgarischen Truppen der Erfolg gegen Serbien, das Kompensation für die territoriale Vergrößerung Bulgariens suchte. In diesem Konflikt, bekannt in der bulgarischen Historiographie als der »Krieg der [serbischen] Generäle gegen die [bulgarischen] Hauptleute«, standen sich ungleiche

12 Vgl. Martin Kröger, Das europäische System »auf des Messers Schneide«. Die Kriegsgefahr der Jahre 1885-1887. In: Vermiedene Kriege. Deeskalation von Konflikten der Großmächte zwischen Krimkrieg und Erstem Weltkrieg 1865-1914, hrsg. von Jost Dülffer, Martin Kröger und Rolf-Harald Wippich, München 1997, S. 369-408, hier S. 373-387.

13 Vgl. Avantjury russkogo zarisma v Bolgarii. Sbornik dokumentov, hrsg. von Pavel Pavlovič, Moskau 1935, im Vorwort, S. 16 [Übersetzung durch die Autorin].

14 Zum Begriff und politischen Konzept des Panslawismus siehe Lars Karl, Adamantios Skordos, Panslawismus. In: European History Online (EGO), hrsg. von dem Leibniz-Institut für Europäische Geschichte (IEG), Mainz 2013-06-06. URL: http://ieg-ego.eu/de/threads/transnationale-bewegungen-und-organisationen/pan-ideologien/lars-karl-adamantios-skordos-panslawismus (5. August 2015). Der russische Panslawismus sah Russland als hegemoniale Macht in einem zukünftigen allslawischen Reich.

15 Vgl. Pavlovič, Avantjury (wie Anm. 13), im Vorwort, S. 16.

16 Zur Operationsgeschichte des Serbisch-bulgarischen Krieges vom 02.-16.11. ${ }^{\text {jul }} 1885$ siehe Istorija na srăbsko-bălgarskata vojna, Sofia 1925. Alle Datenangaben im Text erfolgen nach der julianischen Zeitrechnung, die in Bulgarien bis 1916 gebräuchlich war. 
Kräfte gegenüber. Russland hatte, als Antwort auf den von ihm missbilligten Alleingang Bulgariens, seine Offiziere aus dem bulgarischen Heer abgezogen. Der höchste Dienstgrad eines bulgarischen Offiziers war der eines Majors, die Mehrheit der Kommandeure setzte sich aus Hauptleuten und Subalternoffizieren zusammen. Dieser Krieg wurde zur Geburtsstunde des bulgarischen Offizierkorps ${ }^{17}$, die Emanzipation vom Kult um die russischen Befreier konnte jedoch nicht komplett vollzogen werden.

Die Vereinigung mit Ost-Rumelien und der Erfolg gegen Serbien steigerten Battenbergs Popularität bei der Bevölkerung, bei den nationalistisch gesinnten Politikern und in der Armee. Russland dagegen befürchtete das Schwinden seines Einflusses in Bulgarien zugunsten Österreich-Ungarns und Deutschlands. Die führenden politischen Kreise in Russland mit Zar Alexander III. an ihrer Spitze sahen Battenberg als die Galionsfigur der bulgarischen Bestrebungen um eine politische Eigenständigkeit an. Für sie war er fortan ein Gegner, den es mit allen Mitteln zu bekämpfen galt ${ }^{18}$.

Auf den militärischen Erfolg gegen Serbien folgten die ersten Beförderungen bulgarischer Offiziere und damit auch die ersten verletzenden Zurücksetzungen derjenigen, die nicht ausgezeichnet wurden. So wurde der Hauptmann Anastas Benderev, später einer der Anführer der Verschwörung gegen Battenberg, übergangen. Der Korrespondent der Moskovskie vedomosti in Sofia meldete, es hätte Unzufriedenheit unter den Offizieren gegeben, die Favoriten des Fürsten wären unverdient befördert worden ${ }^{19}$. »In der Armee wird offen von ihrer Teilung in zwei Parteien gesprochen - prorussische und profürstliche ${ }^{20} . \ll$, stellte er fest.

Diese gespaltene Loyalität des bulgarischen Militärs bildete den Ausgangspunkt für die Verschwörung einer Gruppe von Offizieren gegen den bulgarischen Fürsten und deutschen Prinzen Alexander von Battenberg, welche im Folgenden analysiert wird. Dabei wird der Versuch unternommen, die Rolle des geheimen Netzwerkes im Militär und im Staatsleben Bulgariens herauszuarbeiten und die Wechselwirkung von militärischen Strukturen und Untergrundpraktiken zu beleuchten. Nach einem Überblick über die Forschung und Quellenlage zum Thema werden die Ereignisse, die Verschwörung gegen Battenberg von 1886 und der Putschversuch gegen die Regenten im Februar 1887, im komplexen innen- wie außenpolitischen Kontext und unter Berücksichtigung der persönlichen Hintergründe wie auch der politischen Motivation der Verschwörer untersucht. Nachfolgend werden in einer Synthese spezifische Aspekte des in diesen Verschwörungen agierenden geheimen Netzwerks herausgearbeitet und dessen Struktur, die Gruppenkohäsionsmechanismen sowie die Kommunikations- und Geheimhaltungspraktiken analysiert. Ein besonde-

17 Über das bulgarische Offizierskorps im Serbisch-Bulgarischen Krieg vgl. Svetlozar Nedev, Komandvaneto na bălgarskata vojska prez vojnite za nacionalno obedinenie 1885, 1912, 1913, 1915-1918, Sofia 1993.

18 Vgl. Pavlovič, Avantjury (wie Anm. 13), im Vorwort, S. 13.

19 Vgl. Simeon Radev, Stroiteli na săvremenna Bălgarija. Bd. 1: Caruvanieto na kn. Aleksandra 1879-1886, Sofia 1910, Bd. 2: Regentstvoto, Sofia 1911, hier Bd. 1, S. 736.

20 Vgl. ebd., Bd. 1, S. 737 [Übersetzung durch die Autorin]. 
res Augenmerk richtet sich dabei auf die Praktiken, welche die Verschwörer mit der Tradition früherer geheimer Netzwerke und Organisationen verbanden. Der Beitrag schließt mit einer Zusammenfassung, in der die gewonnenen Erkenntnisse zu einem Gesamtbild zusammengefügt werden, um anhand der ausgewählten Fallbeispiele die komplexe Problematik der Beteiligung militärischer Geheimnetzwerke am politischen Leben des bulgarischen Staates, die relevanten Verflechtungen und Einflussfaktoren sowie Auswirkungen zu verdeutlichen.

\section{Forschungsstand und Quellenlage}

Die ersten der Verschwörung gegen Alexander von Battenberg gewidmeten Veröffentlichungen stammen aus der Feder von Zeitzeugen, Politikern, Militärs, Befürwortern sowie Gegnern der Revolte. Die zeitgenössischen Publikationen haben meistens einen politisch tendenziösen Charakter, exemplarisch die Schrift des Journalisten und Battenberg-Unterstützers Zachari Stojanov »Koi sa vinovnicite na 9-tij Avgust? «[Wer sind die Schuldigen am 9. August?] ${ }^{21}$.

Alexander von Battenberg entstammte einer deutschen Adelsfamilie, die mit dem russischen Zarenhaus und der britischen Königsfamilie verschwägert war. Ein Jahr vor der Verschwörung hatte er mit Erfolg die bulgarischen Truppen im Serbisch-Bulgarischen Krieg befehligt. Entsprechend groß war das Interesse an seiner Person im westlichen Europa, vor allem in Deutschland. Der Korrespondent der Kölnischen Zeitung, Arthur von $\mathrm{Huhn}^{22}$, veröffentlichte bereits 1886 eine Darstellung der Ereignisse mit dem Titel »Aus bulgarischer Sturmzeit $\ll^{23}$. Diese wurde zeitnah ins Englische und ins Bulgarische übersetzt $^{24}$. Von Huhn galt als Kenner Bulgariens, berichtete er doch bereits im Russisch-Türkischen Krieg 1877/78 von den Kampfhandlungen an der Donaufront. 1886 publizierte er»Der KampfderBulgarenumihre Nationaleinheit « ${ }^{25}$. Auch wenn von Huhn in seinen Kommentaren gelegentlich polemisch wirkt und eindeutig Battenberg freundlich gegenübersteht, so ist seine Darstellung detailreich und zumindest in der Beschreibung des Ereignisablaufs sehr genau. Das ist vor allem darauf zurückzuführen, dass er einerseits viele unmittelbar involvierte Offiziere und Politiker persönlich kannte und Informationen aus

21 Zachari Stojanov, Koi sa vinovnicite na 9-tij Avgust?, Sofia 1886.

22 Überblick der Publikationen Arthur von Huhns über Bulgarien bei Dietmar Endler, Ein neues Land - Was man Ende des 19. Jahrhunderts in Deutschland über Bulgarien lesen konnte. In: Forum: Bulgarien. Deutsch-bulgarischer Kultur- und Wissenschaftstransfer, hrsg. von Helmut Schaller und Rumjana Zlatanova, Berlin 2013, S. 79-101, hier S. 83-86.

23 Von Huhn (wie Anm. 11).

24 Arthur von Huhn, The Kidnapping of Prince Alexander of Battenberg. His Return to Bulgaria and Subsequent Abdication, London 1887; Artur fon Chun, Iz bălgarskite burni vremena, Russe 1890 .

25 Arthur von Huhn, Der Kampf der Bulgaren um ihre Nationaleinheit. Politisch-militärische Geschichte der Bulgarisch-Rumelischen Ereignisse im Jahre 1885, Leipzig 1886. 
erster Hand beziehen, andererseits die Ereignisse teilweise vor Ort verfolgen konnte. Die Erinnerungen Richard von Machs, der zum Zeitpunkt der Verschwörung Kommandeur einer bulgarischen Brigade war, und jene des Hofgeistlichen Adolf Koch stellen weitere Zeugnisse aus deutscher Feder dar ${ }^{26}$. Auf russischer Seite bieten die Erinnerungen des Diplomaten Jurij Karcov einen Überblick über die innen- wie außenpolitischen Ereignisse in Bezug auf die Verschwörung ${ }^{27}$.

Spätere Studien - hier ist insbesondere das 1910/11 publizierte Werk des bulgarischen Historikers und Diplomaten Simeon Radev »Stroiteli na săvremenna Bălgarija « [Begründer des modernen Bulgariens] ${ }^{28} \mathrm{zu}$ nennen zeichnen ebenfalls detailreich den Ereignisablauf von 1886/87 nach. Radev hatte Zugang zu parlamentarischen Protokollen, zum Archiv des bulgarischen Außenministeriums, zu privaten Nachlässen und Korrespondenzen. Zudem befragte er Zeitzeugen, Politiker wie Militärs, unter ihnen Anastas Benderev, Anführer der Verschwörer. Das Buch Radevs wurde in der sozialistischen Ära 1944-1989 weitgehend marginalisiert, da seine Darstellung der Ereignisse der offiziellen ideologisierten Geschichtsschreibung widersprach. Erst 1990 wurde eine überarbeitete Auflage veröffentlicht, auf welche sich die Nachzeichnung des Ereignisablaufs in dem vorliegenden Beitrag stützt ${ }^{29}$.

Abhandlungen über die Regierungszeit Battenbergs wurden zumeist aus dem Anlass eines Jahrestages (Geburt, Tod, Thronbesteigung) veröffentlicht. Bis in die erste Hälfte der 1940er Jahre schlugen sie einen eher positiven Ton an, zumal die Bündnispolitik Bulgarien in den beiden Weltkriegen an Deutschland band ${ }^{30}$. In diesem Kontext erschien »Bulgariens Befreiung und staatliche Entwicklung unter seinem ersten Fürsten « von Alois Hajek ${ }^{31}$.

In der Zeit des totalitären kommunistischen Regimes 1944-1989 stieg das Interesse an den russophilen Verschwörern, deren Motive und Handlungen im Kontext der engen sowjetisch-bulgarischen Beziehungen gedeutet wur-

26 Vgl. Adolf Koch, Fürst Alexander von Bulgarien. Mittheilungen aus seinem Leben und seiner Regierung nach persönlichen Erinnerungen, Darmstadt 1887; Richard von Mach, Elf Jahre Balkan. Erinnerungen eines preussischen Officiers aus den Jahren 1876-1887, Breslau 1889; ders., Aus bewegter Balkanzeit, 1879-1918, Berlin 1928.

27 Jurij Karcov, Sem< let na Bližnem Vostoke 1879-1886. Vospominanija političeskie i ličnye, Sankt Petersburg 1906.

28 Radev, Stroiteli (wie Anm. 19).

29 Die Beschreibung Radevs stimmt mit der von Arthur von Huhn überein, obwohl beide unabhängig voneinander und mit großem zeitlichem Abstand entstanden sind. Es ist nicht sicher, ob Radev das Buch von Huhns (Aus bulgarischer Sturmzeit, 1886) kannte, obwohl er in seinem Werk eine frühere Publikation (Der Kampf der Bulgaren um ihre Nationaleinheit, 1886) desselben zitiert.

30 Vgl. Krăstjo Mirski, Posreštaneto na părvija bălgarski knjaz Aleksandăr Batenberg v gr. Varna pri vstăpvaneto mu v novosăzdadenoto Knjažestvo Bălgarija, Varna 1930; Danail Kacev-Burski, Knjaz Aleksandăr Batenberg: Kratki životopisni i văzpomenatelni beležki po slučaj 50 god. ot Săedinenieto na Južna i Severna Bălgarija i velikija pobeden podvig na bălgarskata armija nad srăbskata prez 1885, Sofia 1933; Petko Peev, Knjaz Aleksandăr Batenberg: Glavnokomanduvašt 1885, Sofia 1939.

31 Alois Hajek, Bulgariens Befreiung und staatliche Entwicklung unter seinem ersten Fürsten, München u. a. 1939. 
den ${ }^{32}$. Hingegen wurden die Person und das Wirken Battenbergs im Einklang mit dem ideologischen Paradigma einheitlich negativ beschrieben ${ }^{33}$.

In den 1950er und 1960er Jahren entstand, vorerst als Manuskript, das um Objektivität bemühte Werk des Jura-Professors und Diplomaten Georgi Genov zur bulgarischen Politik- und Diplomatiegeschichte. Band 10, veröffentlicht erst 2011, widmet sich ausschließlich der innen- und außenpolitischen Krise von $1886 / 87^{34}$.

Unter den neuesten Publikationen ist die Studie von Georgi Markov »Pokušenija, nasilie i politika v Bălgarija 1878-1944 « [Attentate, Gewalt und Politik in Bulgarien 1878-1944], die unter anderem die Revolte von 1887 thematisiert, zu erwähnen ${ }^{35}$. Der 2012 veröffentlichte Beitrag von Rosica Angelova bringt zwar die Ausbildung bulgarischer Offiziere in Russland als wahrscheinlichen Einflussfaktor mit ihrer Teilnahme an dem Putsch gegen Battenberg in Verbindung, lenkt aber den Blick überwiegend auf die Auswirkung der Beteiligung auf die militärische Laufbahn der Offiziere ${ }^{36}$.

Insgesamt legen alle Publikationen den Fokus auf die innenpolitische Problematik, auf die Person Battenbergs, auf den Handlungsablauf am 9. August 1886 oder sind einzelnen Verschwörern mit biographischem Schwerpunkt gewidmet. Die Besonderheiten des geheimen Netzwerks als komplexer Struktur, der Hintergrund der führenden Mitglieder als Untergrundkämpfer sowie die Verflechtung von tradierten Erfahrungen und Praktiken mit dem militärischen Status der Verschwörer blieben bisher weitgehend unbeachtet.

$\mathrm{Zu}$ den wichtigsten Editionen relevanter Archivmaterialien gehören die Auswahl russischsprachiger Dokumente aus den Beständen des russischen Außenministeriums, darunter die Korrespondenz zwischen dem russischen Außenminister Giers und dem russischen diplomatischen Agenten in Bulgarien Bogdanow $^{37}$, sowie die Sammlungen bulgarischer und österreichisch-ungarischer diplomatischer Dokumente, insbesondere die Korrespondenz zwischen dem österreichisch-ungarischen Diplomaten und Außenminister Graf Kálnoky und dem k.u.k. Diplomatischen Agenten in Sofia Rüdiger von Biegeleben ${ }^{38}$. Darüber hinaus sind die Nachlässe von Stefan Stambolov, Zachari Stojanov,

32 Vgl. Vasil Popov, General Radko Dimitriev - zabeležitelen bălgarski pălkovodec. Biografični beležki, Sofia 1956 (= Voennoistoričeski sbornik, Heft 2, S. 82-106); Ivan Drenski, General Radko Dimitriev, Sofia 1962; Panajot Panajotov, Bălgari - oficeri ot starata ruska armija i săvetskata vlast, Sofia 1977 (= Voennoistoričeski sbornik, Heft 4, S. 26-35).

33 Exemplarisch Michail Ralev, Za družbata s ruskija narod: Borbata na bălgarskite voini sreštu diktaturata na Batenberg i Stambolov 1886-1887, Sofia 1958.

34 Vgl. Georgi Genov, Političeska i diplomatičeska istorija na Bălgarija, Bd. 10: Prevratăt v Sofija na 9 avgust 1886 g. Kontraprevratăt v Plovdiv, Sofia 2011.

35 Vgl. Georgi Markov, Pokušenija, nasilie i politika v Bălgarija 1878-1944, Sofia 2003.

36 Vgl. Rosica Angelova, Bălgarski văzpitanici na Odeskoto pehotno junkersko učilište 1865-1918 - profesionalna realizacija i političeska sădba, Šumen 2012 (= Istorikii, 5), S. 135-167, hier S. 162-167.

37 Pavlovič, Avantjury (wie Anm. 13), übersetzt ins Bulgarische von Svetlana Draganova (Avantjurite na ruskija carisăm v Bălgarija, Sofia 1991). Im Beitrag wird aus dem russischen Original zitiert.

38 Bălgarija v avstro-ungarskite diplomatičeski dokumenti 1879-1885, hrsg. von Cvetana Todorova u. a., Sofia 1993; Izvori za bălgarskata istorija, hrsg. von Penka Kostadinova und Milen Kumanov, Sofia 1994. 
Anastas Benderev, Alexander Battenberg und Sava Mutkurov im Bulgarischen Historischen Archiv der Nationalbibliothek »Hl.Hl. Kyrill und Method « in Sofia sowie Bestände des Militärarchivs in Tărnovo von Bedeutung ${ }^{39}$.

\section{Die Verschwörung gegen Battenberg}

Der Putsch russophiler Offiziere gegen Battenberg wurde mit russischer Hilfe vorbereitet. Nahezu alle Verschwörer hatten als Freiwillige im RussischTürkischen Krieg 1877/78 unter russischem Kommando gekämpft. Sämtliche führenden Köpfe der Verschwörung hatten zwischen 1881 und 1885 die Militärakademie in Sankt Petersburg besucht und abgeschlossen und unterhielten enge Kontakte zu russischen politischen und militärischen Entscheidungsträgern $^{40}$. Einige verband die Überzeugung, dass Bulgariens Existenz ohne russische Protektion gefährdet, ja undenkbar wäre. Andere hegten Karriereambitionen, deren Erfüllung sie nur im mächtigen russischen Heer als realisierbar ansahen oder fühlten sich bei Beförderungen und Auszeichnungen übergangen $^{41}$. Agenten des Asiatischen Departements im russischen Außenministerium sowie der russische Militärattaché in Sofia Vsevolod Sacharow bemühten sich, diese für die Verschwörung zu gewinnen und stellten ihnen Karrieremöglichkeiten in der russischen Armee oder finanzielle Unterstützung in Aussicht ${ }^{42}$. Dem ehemaligen Leiter der Militärschule in Sofia und nunmehrigen russischen Militärattaché Sacharow, der engen Kontakt zu seinen früheren Untergebenen pflegte, waren die Pläne der Verschwörer bekannt ${ }^{43}$.

Das Netzwerk der Verschwörer blieb verhältnismäßig klein. Die Anführer rekrutierten Sympathisanten, mit denen sie jahrelange vertraute Beziehungen verbanden - Freundschaften aus Studienzeiten, gemeinsame Erfahrungen in Untergrundorganisationen. Viele von ihnen hatten vor 1877/78 den sogenannten geheimen Revolutionskomitees angehört - Untergrundvereinigungen, die einen bewaffneten Aufstand gegen die osmanischen Herrscher vorbereiteten. So waren der Vize-Kriegsminister Benderev ${ }^{44}$ und der Abteilungsleiter im

39 Bulgarisches Historisches Archiv in der Nationalbibliothek »H1.Hl. Kyrill und Method « in Sofia (BIA-NBKM), Fonds 63 Stefan Stambolov, 100 Zachari Stojanov, 262 Anastas Benderev, 271 Alexander von Battenberg, 342 Sava Mutkurov; Staatliches Militärhistorisches Archiv, Tărnovo (DVIA), Fonds 1 und 7 Kriegsministerium/Ministerstvo na vojnata, Fond 1521 Offiziersschule/Sofijsko voenno učilište, Fond 298 Militärgericht Sofia/Sofijski voenno-polevi săd, Fond 991 Militärgericht Russe/Rusenski voenen săd.

40 Vgl. Radev, Stroiteli (wie Anm. 19), Bd. 1, S. 737.

${ }^{41}$ Vgl. ebd., S. $738 \mathrm{f}$.

42 Vgl. Pavlovič, Avantjury (wie Anm. 13), im Vorwort, S. 16; Radev, Stroiteli (wie Anm. 19), Bd. 1, S. 749-751.

43 Vgl. Bericht des russischen Militärattaché in Sofia Sacharow an den Generalstabschef Obrutschew vom 31.08.jul. 1886 in: Pavlovič, Avantjury (wie Anm. 13), S. 20.

${ }_{44}$ Ausführlich über Anastas Benderev als Militär und politischer Akteur siehe Marija Šalaverova, Anastas Benderev kato voenno-političeska ličnost, Sofia 1980 (= Izvestija na Instituta po voenna istorija, 29), S. 127-159. 
Kriegsministerium, Radko Dimitriev ${ }^{45}$, vor 1877 erfahrene Konspiratoren und führende Figuren in den geheimen Revolutionskomitees. Die geringe Zahl der tatsächlich Eingeweihten - anfangs nicht mehr als zehn Offiziere - der enge Kontakt sowie die Übung im Aufbau geheimer Netzwerke erlaubten es in der kurzen Zeit von nur etwa drei Monaten, die Umsturzpläne zum Abschluss zu bringen. Zunächst standen lediglich zwei der zehn bulgarischen Regimenter unter der Kontrolle der Verschwörer. Sie verschafften sich jedoch einen Vorteil, indem sie ihre hohen Stellungen im Kriegsministerium nutzten, um - unter dem Vorwand einer bevorstehenden erneuten Aggression Serbiens - Truppen umzugruppieren: die Battenberg loyalen Einheiten kamen an die serbische Grenze, während die von ihren Mitwissern befehligten Regimenter in die Hauptstadt verlegt wurden. Für ihre Sache konnten sie auch den Leiter der Offiziersschule Major Petăr Gruev gewinnen ${ }^{46}$, was ihnen die Unterstützung der Kadetten sicherte. Gruev, selbst kein Gegner Battenbergs, vertrat die Auffassung, dass allein Russland Bulgarien vor einer militärischen Katastrophe in dem vermeintlichen neuen Krieg bewahren könnte. Den Sturz Battenbergs sah er als Notlösung, die eine gefährliche Krisensituation für das Land beenden sollte.

Die Verschwörer bemühten sich auch um politische Unterstützung - der Ministerpräsident Petko Karavelov und der Kriegsminister Konstantin Nikiforov sollten mit einbezogen werden. Nach erstmaligen Gesprächen lehnten beide eine aktive Beteiligung ab, ergriffen jedoch auch keinerlei Maßnahmen gegen die Verschwörer"

Ziel der Gruppe war es, neben der Absetzung Battenbergs, Bulgarien wieder an Russland anzunähern. Unter den Verschwörern hatte Radko Dimitriev eine klare Vision für die politische Zukunft des Landes - als russisches Protektorat. »Wir wollten «, schrieb Dimitriev nach dem Putsch in Moskovskie vedomosti, »dass Russland einen Kriegsminister, Divisions- und Regimentskommandeure entsendet, welche die höchsten Posten im Land besetzen sollten « ${ }^{48}$. Dimitriev war für seine glühende Russophilie bekannt und dem Panslawismus nicht fremd, seine Loyalität galt den »Bratuschki«, den russischen Waffenbrüdern und dem »Zar-Osvoboditel «, dem Zaren-Befreier und seinem Nachfolger Alexander III..$^{49}$.

Da den Verschwörern die Popularität Battenbergs unter den Offizieren und Soldaten bewusst war, wurden nur einzelne Sympathisanten über die Ziele der Gruppe in Kenntnis gesetzt. Diese sollten mit ihrem Ehrenwort die Bewahrung des Geheimnisses garantieren ${ }^{50}$. Den Kadetten der Militärschule und den Soldaten der für die Durchführung des Umsturzes vorgesehenen Regimenter wurde erst einige Stunden vor der Aktion der wahre Grund für ihren Einsatz eröffnet ${ }^{51}$. Zuvor wurde ihnen suggeriert, ein serbischer Angriff stünde unmit-

5 Vgl. Drenski, General (wie Anm. 32) S. 9 f.

46 Vgl. Radev, Stroiteli (wie Anm. 19), Bd. 1, S. 749.

47 Vgl. ebd., S. 739.

48 Vgl. ebd., S. 738 [Übersetzung durch die Autorin].

49 Vgl. ebd., S. $738 \mathrm{f}$.

50 Vgl. ebd., S. 754.

51 Vgl. ebd., S. 765. 
telbar bevor, was eine erhöhte Truppenkonzentration zum Schutz der Hauptstadt erforderlich mache. Nach der Enthüllung ihrer wahren Ziele begründeten die Verschwörer ihr Handeln damit, Russland würde, solange Battenberg auf dem Thron bleibe, Bulgarien nicht vor der serbischen Aggression schützen wollen, und appellierten an den Patriotismus der Offiziere. Ihnen wurde eine breite Unterstützung der politischen Entscheidungsträger, der Zivilbevölkerung und der Soldaten in mehreren Truppeneinheiten zugesichert. Trotzdem ließen sich die meisten Offiziere nur unwillig auf den Umsturz ein. Benderev bezeugte später im Verhör, dass die persönlichen Sympathien der Offiziere eindeutig auf der Seite Battenbergs gelegen hätten. Allein die Überlegung, dass ohne seinen Rücktritt Bulgarien große Gefahren drohten, konnte sie zum Eingreifen bewegen: An der Verschwörung beteiligte Offiziere hatten sich auf Aufklärungsreise begeben und von einer - erfundenen - Verlegung serbischer Truppen an die bulgarische Grenze berichtet.

Der russische Militärattaché Sacharow indes versicherte über Benderev, dass, unter der Bedingung einer Absetzung Alexander von Battenbergs, Russland einen erneuten Angriff Serbiens nicht zulassen würde ${ }^{52}$. Auch die Autorität Benderevs als Vize-Kriegsminister zeigte Wirkung.

Die Geheimhaltung ließ in den Tagen vor der Aktion nach. Vermutlich wähnten sich die Verschwörer unter russischer Protektion in Sicherheit. Es kursierten Gerüchte, die sogar Battenberg zugetragen wurden, der sie aber ignorierte. Ihm war die Animosität Dimitrievs und Benderevs zwar bekannt, jedoch schloss er deren Loyalitätsbruch aus ${ }^{53}$.

Zunächst zogen die Verschwörer die Ermordung Battenbergs in Betracht, verwarfen aber den Plan, da sich der russische diplomatische Agent in Sofia, Bogdanow, dagegen ausgesprochen hatte. Stattdessen forderte er, dass Battenberg abgesetzt, aus dem Land geführt und den Russen übergeben werden sollte. Der russische Diplomat stand in engem Kontakt mit den Verschwörern und wurde über ihr Vorhaben genau informiert; ein Telegramm ${ }^{54}$ an den russischen Außenminister Nikolai de Giers, entsandt einen Tag vor dem Umsturz, enthielt Details über deren Absichten und Pläne.

In der Nacht auf den 9. August ${ }^{\text {jul. }} 1886$ wurde die Hauptstadt Sofia von dem in die Verschwörung eingeweihten Infanterieregiment und den Kadetten der Militärschule besetzt und der Palast gestürmt. Alexander von Battenberg wurde gefangen genommen, gezwungen eine Abdankungserklärung zu unterschreiben und unter bewaffneter Begleitung über die Donau nach Russland befördert. Die Verschwörer ernannten eine Interimsregierung und bemühten sich mit mäßigem Erfolg, die Garnisonen, die nicht an dem Umsturz beteiligt worden waren, auf diese zu vereidigen. Sie fanden geringe Unterstützung innerhalb der politischen Elite sowie in der breiten Öffentlichkeit, zu der sie kaum den Kontakt gesucht hatten. Bereits am folgenden Tag erklärten sich einige Garnisonskommandanten gegen die Verschwörung. Den Gegnern des

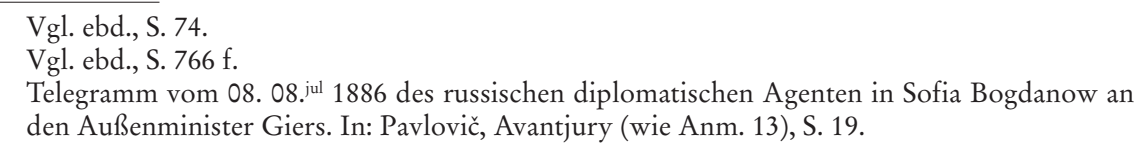


Umsturzes unter der Führung des Parlamentspräsidenten Stefan Stambolov ${ }^{55}$ gelang es daraufhin binnen weniger Tage, die Battenberg loyalen Truppen auf ihre Seite zu ziehen und den Fürsten vorübergehend zurück ins Land zu holen ${ }^{56}$. Die führenden Köpfe der Verschwörung konnten sich über die Donau nach Rumänien retten.

Alexander von Battenberg dankte am 26. August ${ }^{\text {jul. }} 1886$ freiwillig ab, da er es trotz der Unterstützung im Land als unmöglich ansah, sich fernerhin gegen den Willen Russlands zu behaupten ${ }^{57}$. Er bestimmte den Ministerpräsidenten Karavelov, den Parlamentspräsidenten Stambolov und den Kommandanten einer loyalen Garnison zu Regenten ${ }^{58}$.

Die Sanktionen gegen die an dem Umsturz beteiligten Offiziere und Soldaten fielen verhältnismäßig mild aus. Die beteiligten Regimenter wurden aufgelöst, die Offiziere degradiert oder vorübergehend vom Dienst suspendiert. Einige wurden verhaftet, auf russischen Druck jedoch wieder freigelassen. Andere involvierte Militärs und zivile Unterstützer der Verschwörung fürchteten Repressalien und gingen ins Exil nach Rumänien, Serbien, Russland und ins Osmanische Reich ${ }^{59}$.

\section{Russischer Druck nach der Abdankung Battenbergs}

Russland suchte die instabile innere Lage und die außenpolitische Isolation Bulgariens zu nutzen und setzte diplomatische Mittel wie militärische Machtdemonstrationen ${ }^{60}$ ein, um die von Battenberg eingesetzten Regenten zur politischen Umkehr zu bewegen. Die russischen Diplomaten in Sofia und insbesondere in Odessa konnten auf ein gut ausgebautes Netzwerk zurückgreifen, da viele bulgarische Offiziere in der dortigen Militärschule für Offiziere der Infanterie ausgebildet worden waren ${ }^{61}$. So berichtete der diplomatische Vertreter in Sofia an den Außenminister Giers vom Wunsch eines bulgarischen Of-

55 Über die Person und das politische Wirken des späteren Premierminister Stefan Stambolov siehe Duncan M. Perry, Stefan Stambolov and the Emergence of Modern Bulgaria 1870-1895, Durham NC 1993.

56 Siehe Proklamation des Parlamentspräsidenten Setfan Stambolov an das bulgarische Volk vom 16.08. jul. 1886 und Proklamation Alexander I. an das bulgarische Volk vom 17. 08 jul. 1886. In: Kostadinova, Izvori (wie Anm. 38), S. 298 f.

57 Siehe Telegramm des bulgarischen Fürsten Alexander I. an den russischen Zaren Alexander III. und Telegramm Alexander III. an Alexander I. In: Kostadinova, Izvori (wie Anm. 38), S. 299 f.

58 Siehe Manifest Alexander I. an das bulgarische Volk vom 26.08. jul. 1886. In: Kostadinova, Izvori (wie Anm. 38), S. 300 f.

59 Vgl. Radoslav Popov, Bălgarija na krăstopăt. Regenstvoto 1886-1887, Sofia 1991, S. 38-88, 131134.

60 Russische Kriegsschiffe liefen im Oktober ${ }^{\text {jul. }} 1886$ in den Hafen von Varna, vgl. Radev, Stroiteli (wie Anm. 19), Bd. 2, S. 396 f.

${ }_{61} \mathrm{Zu}$ den bulgarischen Offizieren, die ihre militärische Ausbildung in Odessa erhalten haben und deren Rolle in den Ereignissen vom August ${ }^{\text {jul }} 1886$ und Februar ${ }^{\text {jul }} 1887$ siehe Angelova, Bălgarski văzpitanici (wie Anm. 36) S. 135-167. 
fiziers, während des Vorgehens gegen die Regenten eine russische Uniform tragen zu dürfen, da er früher in der russischen Armee gedient habe ${ }^{62}$.

Im Oktober 1886 brach eine Militärrevolte in der prorussischen Garnison der Hafenstadt Burgas aus, wurde aber nach wenigen Tagen von den regierungstreuen Truppen beendet. Ein Teil der Anstifter rettete sich ins russische Konsulat, andere flüchteten nach Konstantinopel.

Inzwischen traf der russische Militärkommissar General Nikolai von Kaulbars in Bulgarien ein, um die russischen Interessen nach der Abdankung Battenbergs zu vertreten ${ }^{63}$. Russland wollte keine Besetzung Bulgariens, da es sich nicht für einen neuen Krieg auf dem Balkan gerüstet sah und eine ungünstige Einmischung Großbritanniens in der »Orientalischen Frage « befürchtete. Andererseits strebte Russland eine vollständige Kontrolle über Außenpolitik und Armee Bulgariens an, um diese in einem zukünftigen Interessenkonflikt zu seinen Gunsten einsetzen zu können ${ }^{64}$.

Die Mission Kaulbars scheiterte an der Weigerung der Regenten und der nationalistisch gestimmten Offiziere, den russischen Forderungen nach Rücktritt der Regierung zu folgen. So berichtete er an Giers, dass »der Widerstand gegen uns in großen Teilen der bulgarischen Armee ausschließlich auf dem Widerstand der Offiziere beruht « ${ }^{65}$. Des Weiteren erwähnte er die Möglichkeit, durch einen schnellen, von einer kleinen russischen Einheit durchzuführenden Streich, die bulgarischen Kommandostrukturen in der Armee durch russische zu ersetzen: »Selbstverständlich können wir diesen Plan nur dann verwirklichen, wenn sich Europa auf eine Besetzung einlässt; und sollte dies eintreten, wäre es besser viel mehr Truppen zu entsenden ${ }^{66}$. Der Plan wurde von Giers abgelehnt, eine Besetzung sah er als die »äußerste Notmaßnahme ${ }^{67}$." Kaulbars wurde zurückbeordert, im November 1886 brach Russland die diplomatischen Beziehungen zu Bulgarien ab.

Die führenden Figuren der Verschwörung scheinen durch innere Gegensätze geprägt worden zu sein. Zum einen spielte ihre Vergangenheit als Mitglieder eines geheimen Netzwerkes, in dem sie erste Erfahrungen im Untergrundkampf und in der Geheimhaltung sammeln konnten, eine Rolle. Zum anderen bauten sie eine militärische Karriere auf und wurden von Rebellen gegen staatliche Gewalt zu legitimen Schützern des Staates, um dann sich wiederum gegen den Staat aufzulehnen. Einerseits waren sie - die früheren Kämpfer gegen das Osmanische Reich - bulgarische Patrioten. Andererseits fühlten sie sich ihren russischen Ausbildern und Vorgesetzten verbunden und wurden zu Instrumenten russischer Machtpolitik in Bulgarien. Ihre Loyalität galt Russland und nicht dem bulgarischen Fürsten; diesen sahen sie nicht als ihren Monarchen und obersten Befehlshaber, sondern als ersetzbare Marionette der europäischen

62 Vgl. Telegramm des russischen Botschafters in Sofia an Giers vom 05.10. jul. 1886. In: Pavlovič, Avantjury (wie Anm. 13), S. 28.

63 Über die Mission von Kaulbars< siehe Radev, Stroiteli (wie Anm. 19), Bd. 2, S. 277-308.

${ }^{64}$ Vgl. ebd., S. 384 f.

65 Vgl. Pavlovič, Avantjury (wie Anm. 13), S. 29.

66 Vgl. ebd., S. 30.

67 Vgl. ebd., S. 31. 
Großmächte, welche zur Gefahr für die Allianz mit Russland und hiermit für das Wohl Bulgariens geworden war. In diesem Sinne agierten sie 1886/87 als loyale Konspiratoren - mit ihrer Identität im geheimen Untergrund verwurzelt, ihrem Vaterland und einem fremden Staat zugleich loyal.

\section{Die Revolte gegen die bulgarischen Regenten von 1887}

Die Verschwörung vom 9. Augustjul. 1886 hatte ein halbes Jahr später ein Nachspiel. Benderev und Gruev wurde im Außenministerium in Petersburg Unterstützung für eine weitere Revolte zugesichert, beide begaben sich nach Rumänien $^{68}$. Die gescheiterten Verschwörer organisierten sich in einem Geheimen Revolutionskomitee mit Sitz in Bukarest; einen Ableger gab es in Konstantinopel. Damit signalisierten sie, dass sie sich in der Tradition der früheren Freiheitskämpfer sahen und nutzten zugleich ihnen vertraute Praktiken und Kommunikationskanäle ${ }^{69}$. Mehrere der Verschwörer waren in den Jahren vor der Unabhängigkeit Bulgariens Mitglieder der geheimen Komitees der IRO und konnten auf noch vorhandene Strukturen und Beziehungen zu früheren Weggefährten zurückgreifen. Sie machten sich die nationalistische Aura dieser Widerstandsnetzwerke zu eigen, um an den Patriotismus potentieller Mitstreiter zu appellieren. Dabei bedienten sie sich einer Kodiersprache, deren Vokabular ehemaligen Mitstreitern vertraut war und ihr Vorhaben in die Tradition früherer Unabhängigkeitskämpfer stellte ${ }^{70}$. Nicht alle folgten ihrer Vorstellung von Tradition. Ein Regimentskommandeur äußerte sich dem Komitee gegenüber: »Ich bedaure, dass ihr hier, wo vor zehn Jahren das Komitee für die Unabhängigkeit Bulgariens tätig war, nun für seine Unterwerfung arbeitet ${ }^{71}$ «

Auffallend ist, dass Offiziere, die am Putsch gegen Battenberg beteiligt waren, die aber in ihrer Vergangenheit keine Erfahrung mit geheimen Netzwerken hatten, sich dem Komitee nicht anschlossen, sich hingegen aber alle, die auch früher im Untergrund tätig waren, an der Gründung und dem Ausbau der Strukturen beteiligten. Die Verschwörer hatten außer dem groben Plan, die von ihnen unterwanderten Einheiten für den Staatsstreich einzusetzen, keine konkreten Vorstellungen über ihr weiteres Vorgehen entwickelt. Das Komitee unterhielt enge Beziehungen zu russischen Diplomaten und vermittelte im Land ihre Position zur Krisensituation: »Russland will Bulgarien nicht besetzen, braucht es aber, um Konstantinopel zu erobern. Wenn aber die Bedingungen von Kaulbars nicht erfüllt und die Regenten nicht gestürzt werden, wird eine Besetzung zwingend. Kann die bulgarische Armee den Umsturz vollzie-

\footnotetext{
Vgl. ebd., im Vorwort, S. 18.

Vgl. Radev, Stroiteli (wie Anm. 19), Bd. 2, S. 556 f.

Vgl. ebd., S. 404-406, $557 \mathrm{f}$.

Vgl. ebd., S. 555 [Übersetzung durch die Autorin].
} 
hen, der eine bulgarische Regierung nach dem Willen Russlands etablieren würde ${ }^{72}$ ?

Der Wille Russlands wurde indes von seinen Diplomaten unmissverständlich zum Ausdruck gebracht. Der russische Gesandte in Bukarest Michail Hitrowo, angewiesen von Außenminister Giers, beteiligte sich aktiv an den Umsturzplänen $^{73}$. Giers wünschte, neben der finanziellen Unterstützung für die Verschwörer, auch die minutiöse Beobachtung und Kontrolle ihrer Tätigkeit. Alexander Nelidow, der russische Gesandte in Konstantinopel, organisierte Waffenlieferungen ${ }^{74}$. Die Verschwörer bekamen russische Pässe, was sie im Falle eines Misserfolges vor Strafverfolgung schützen sollte, und ihnen wurden erhebliche Geldsummen zur Verfügung gestellt ${ }^{75}$. Zwei Mitglieder des Komitees wurden sogar vom russischen Zaren empfangen ${ }^{76}$.

Die Verschwörer rechneten damit, dass Russland nach dem erfolgten Staatsstreich die politische Zukunft Bulgariens gestalten würde, bemühten sich daher nicht um politische Unterstützung. Sie suchten nicht einmal den Kontakt zu potentiellen Mitstreitern aus den Reihen der liberalen, prorussischen Opposition im Land. Andererseits aber betrieben sie eifrig Propaganda gegen die Regenten und insbesondere gegen Stefan Stambolov, der selbst ein früheres Mitglied der IRO und des Revolutionskomitees in Bukarest 1874/76 war. Stambolov vermerkte in seinem Tagebuch am 24. Oktober 1886 über die Autoren der Propagandablätter: »Der Katkowismus hat wohl großen Einfluss auf sie gehabt, denn ihre Sprache spiegelt die der Moskovskie vedomosti ${ }^{77}$ «

Die Pläne der Verschwörer waren vage und bauten teilweise auf Wunschdenken auf. Sie hatten vor, Truppen aus mehreren Garnisonstädten gegen die Regierung in einem spontanen Feldzug nach Sofia zu führen. Sie glaubten, über mehr als die Hälfte aller Truppen im Lande verfügen zu können. Die Regenten ihrerseits konnten auf die Unterstützung der Garnisonen im Süden Bulgariens zählen und sahen die Bemühungen von Benderev, Gruev und Dimitriev als einen letzten Versuch, eine politische Wende herbeizuführen. Andererseits fürchteten sie, dass ein sich hinziehender Bürgerkrieg eine Einmischung Russlands und in der Folge eine russische Besetzung bedeuten könnte ${ }^{78}$.

Im Dezember jul. 1886 wurde in Russe von wohlhabenden Bürgern ein weiteres Komitee gegründet, das Verbindungen zu suspendierten Offizieren unterhielt. Sie konnten den Garnisonskommandanten Atanas Uzunov ${ }^{79}$ für sich gewinnen, der wiederum in Kontakt zu dem russischen Gesandten Hitrowo in Bukarest stand ${ }^{80}$. Dieser informierte laufend Giers über die Fortschritte des Komitees. Anfang Februarijul. 1887 berichtete er über ein Treffen mit den Grün-

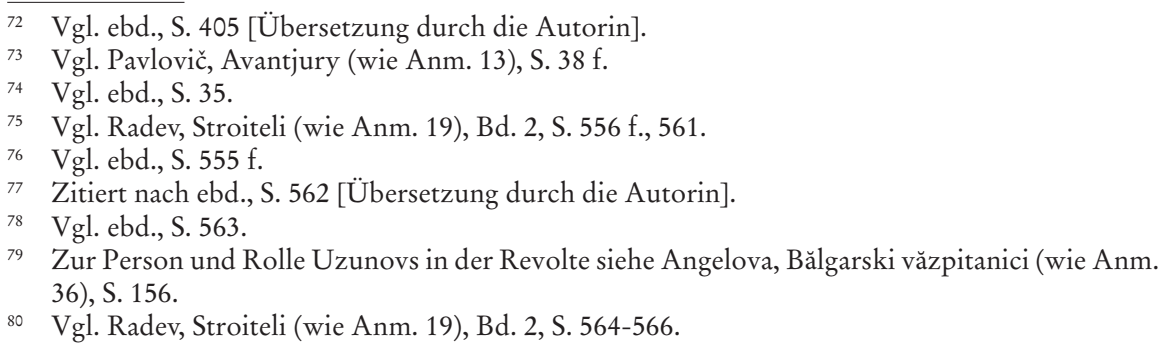


dern des Komitees, Benderev, Dimitriev und Gruev, bei dem eine Geldübergabe stattfand.

Kurz darauf kam es am 16. jul. Februar 1887 zum vorzeitigen Ausbruch der Revolte, da die Regierung Signale erhalten und erste Gegenmaßnahmen wie Versetzungen von verdächtigen Offizieren und verstärkte Grenzkontrollen ergriffen hatte ${ }^{81}$. Bereits an dem Tag informierte Hitrowo über die gesicherten Kommunikationskanäle zwischen den Komitees in Bukarest und Konstantinopel; zwei Tage später entsandte er gar drei Telegramme an Giers. Er berichtete über den Verlauf der Revolte, über finanzielle Hilfe und Munitionslieferungen an die Verschwörer. Um die Kommunikations- und Nachschubwege $\mathrm{zu}$ erleichtern, hatte er sich die wohlwollende Passivität der rumänischen Grenzbehörden gesichert ${ }^{82}$.

Ähnlich wie im August ${ }^{\text {jul }} 1886$ wurde lediglich ein relativ kleiner Kreis Offiziere in die Absichten der Verschwörer eingeweiht. Nur zwei Garnisonen - in Silistra und Russe - beteiligten sich an der Revolte. Beide Garnisonstädte lagen an der Donau, was die Verbindung zum Geheimkomitee, der Zentrale der Verschwörung in Bukarest, erleichterte. Andererseits blieben die Garnisonen im Inneren des Landes im Abseits und waren nach dem Ausbruch der Revolte kaum erreichbar. Die Vorstellung der Verschwörer, dass sich die restlichen Truppeneinheiten zu einer Massenbewegung gegen die Regenten mitreißen lassen würden, entpuppte sich als Chimäre. Auch die städtische Bevölkerung begegnete den Aufrufen zum bewaffneten Aufstand gegen die Regierung ohne Enthusiasmus. Die Bürger von Silistra weigerten sich, eine an den russischen Zar gerichtete Treuebezeugung zu unterschreiben ${ }^{83}$.

Die oberflächliche Vorbereitung, die oft allein auf der Autorität der Kommandeure basierte, hatte zur Folge, dass Unteroffiziere und Soldaten sich gegen ihre Vorgesetzten stellten oder flüchteten. $\mathrm{Zu}$ bewaffneten Auseinandersetzungen mit regierungstreuen Truppen kam es nur in zwei der sieben Garnisonstädte. Die Verschwörung wurde binnen Tagen zerschlagen ${ }^{84}$. Einige Anführer wurden gefasst, verurteilt und hingerichtet, andere flohen nach Russland. Die prominentesten Verschwörer, Dimitriev und Benderev, erhielten nach steiler Karriere in der russischen Armee sogar Generalsposten und befehligten russische Truppen im Ersten Weltkrieg, in den Bulgarien an der Seite der Mittelmächte eintrat.

\section{Merkmale des geheimen Netzwerks von 1886/87}

Das geheime Netzwerk, welches die Verschwörung gegen Battenberg 1886 wie auch jene gegen die Regenten 1887 vorbereitete, hatte eine klare hierarchische

\footnotetext{
Vgl. ebd., S. 567.

Vgl. Pavlovič, Avantjury (wie Anm. 13), S. 40, 42, 43-47.

Vgl. Radev, Stroiteli (wie Anm. 19), Bd. 2, S. 568.

Vgl. Georgi Markov, Pokušenija (wie Anm. 35) S. 16 f.
} 
Struktur. An der Spitze standen Offiziere, die einige der höchsten Positionen im Militär innehatten - der Vize-Kriegsminister Hauptmann Benderev, der Abteilungsleiter im Kriegsministerium Hauptmann Dimitriev und der Leiter der Offiziersschule Major Gruev. Somit konnten sie ungehindert auf Ressourcen und Strukturen des Militärs zurückgreifen, um ihr Vorhaben umzusetzen. Sie standen in engstem Kontakt mit russischen Diplomaten und verfügten über mehr Informationen hinsichtlich der genauen Ziele und der Planung der Verschwörung als ihre eingeweihten Untergebenen.

Die Verschwörung gegen Battenberg war das Resultat des gemeinsamen Agierens russischer Machthaber und rebellierender bulgarischer Militärs. In dieser Hinsicht war sie kein rein bulgarisches und kein rein militärisches Phänomen. Die russischen Diplomaten bildeten eine übergeordnete Gruppe, die die bulgarischen Verschwörer beeinflusste, an den Umsturzplänen mitwirkte und die Revolte finanziell sowie mit Informationen und Waffen entscheidend unterstützte. Darüber hinaus schirmte sie die Verschwörer gegen Strafverfolgung ab. Von Russland erhofften sich die beteiligten bulgarischen Militärs auch nach dem eventuellen Erfolg ihres Unternehmens weiterhin Unterstützung und gegebenenfalls sogar die Besetzung Bulgariens. Somit wären der eigene Übergang in die russische Armee und damit aus ihrer Sicht bessere Karrierechancen für sie offen.

Andererseits agierten die Verschwörer in der konkreten Ausführung des Vorhabens relativ autonom. Sie erarbeiteten Planungsdetails selbstständig, entschieden über Zeitpunkt und Vorgehensweise und berieten zunächst unter sich über eine mögliche Ermordung Battenbergs. Es bleibt unklar, inwiefern die Vorstellungen der Verschwörer mit den russischen Absichten deckungsgleich waren. Beide Seiten beteiligten sich an den Abläufen kooperativ wie kollaborativ, waren dabei aufeinander angewiesen, aber beeinflussten sich auch negativ durch Fehlkalkulationen und Misserfolge.

Die meisten dem Netzwerk angehörenden Militärs teilten eine tief wurzelnde Verbundenheit mit Russland und eine ablehnende Haltung gegenüber Battenberg. Ersteres resultierte aus persönlichen Erfahrungen während der Ausbildung unter russischen Vorgesetzten oder war in paradoxer Weise Folge der patriotischen Überzeugung, dass Bulgarien allein als Satellit des mächtigen slawischen Imperiums bestehen könne. Nicht alle Mitglieder waren Battenbergs Gegner und nicht alle russophilen Offiziere beteiligten sich an der Verschwörung. Auch engagierten sich nicht alle Beteiligten in gleichem Maße oder wurden aktiv, als die Revolte ausbrach. Trotzdem bildeten prorussische Ansichten und auf unterschiedlichen, teilweise persönlichen Motiven beruhende Ressentiments gegenüber dem deutschstämmigen Fürsten die wesentliche Basis für die Inklusion der Gruppe.

In der konkreten Ausführung ihrer Pläne setzten die Anführer Benderev, Dimitriev und Gruev und die an der Verschwörung beteiligten Kommandeure ausschließlich auf ihre Autorität als Vorgesetzte gegenüber ihren Soldaten und den Kadetten der Offiziersschule. Sie waren sich der Loyalität ihrer Untergebenen sicher und rechneten offenbar nicht mit nennenswertem Widerstand seitens der Offiziere und Mannschaften in den involvierten Einheiten. Dies 
erwies sich allerdings als Fehlkalkulation, da Kommandeure und Soldaten wiederholt Zweifel äußerten oder den Gehorsam verweigerten. Vielen bulgarischen Offizieren wurde nach dem Serbisch-Bulgarischen Krieg von 1885 eine rasante Beförderung zuteil, da Russland seine im bulgarischen Heer dienenden Offiziere abgezogen hatte. Sie wünschten keine Annäherung an Russland und nahmen gegenüber den Verschwörern eine ablehnende Haltung ein. Der Versuch der Gruppe, politische Unterstützung für ihr Vorhaben zu sichern, erzielte nur mäßigen Erfolg. Der Ministerpräsident und der Kriegsminister beteiligten sich nicht an der Verschwörung, versuchten aber auch nicht, diese zu vereiteln. Auch wenn die Verschwörer auf russische Unterstützung bauen konnten und zunächst ihr Ziel erreichten, blieb ihr Vorhaben insgesamt unpopulär und scheiterte schließlich am Widerstand innerhalb des Militärs und seitens der politischen Elite sowie an der mangelnden Akzeptanz in der Bevölkerung.

Blickt man auf die Kommunikation und die Wahrung der Geheimhaltung innerhalb des Netzwerkes fällt auf, dass die einzelnen involvierten Garnisonen bzw. Einheiten anfangs koordiniert handelten, danach riss die Kommunikation teilweise oder ganz ab. Während der Aktion konnten die Verschwörer sowie deren Gegner über Kuriere oder über ein telegraphisches Kommunikationssystem miteinander in Verbindung treten. Sie benutzten oftmals Fragen nach privaten Informationen zur Verifikation oder als Erkennungsmerkmal. Obwohl die Gruppe bemüht war, ihre Tätigkeit geheim zu halten und obwohl ihre führenden Köpfe im Falle eines Misserfolges Sanktionen fürchteten, sickerten Gerüchte durch. Das könnte eine Folge der Fehlannahme sein, sie würden auf breite Unterstützung seitens der eigenen Kameraden, der oppositionellen Politiker und letztendlich der Bevölkerung stoßen, die sie für latent russophil erachteten. Ein anderer Faktor, der möglicherweise zur Missachtung der strikten Wahrung des Geheimnisses führte, war die Aussicht auf Straflosigkeit durch die russische Protektion, zumindest für die führenden Verschwörer, die mit russischen Pässen versorgt und denen von russischen Diplomaten Fluchtwege und Asyl ermöglicht wurden.

Schließlich ist im Hinblick auf die Versuche der Verschwörer, die Bevölkerung für ihre Ziele zu gewinnen, und neue Mitglieder zu rekrutieren, herauszustellen, dass diese hier auf Formen der früheren Unabhängigkeitsbewegung zurückgriffen, vor allem aber die bestehenden militärischen Strukturen nutzten. Die geheimen Komitees, welche die Verschwörer gründeten, ähnelten denjenigen der früheren Unabhängigkeitsbewegung. Hierdurch versuchten sie, nationalistisch gesinnten Offizieren und Teilen der Gesellschaft zu imponieren. Diese Komitees boten auch der Bevölkerung eine Beteiligungsform, die - da abgekoppelt von militärischen Strukturen - zugänglich für Zivilisten war. Allerdings hielten sich die Bemühungen der Verschwörer, die breite Öffentlichkeit anzusprechen, eher in Grenzen und brachten nicht den erwünschten $\mathrm{Zu}-$ spruch.

Das Knüpfen von Kontakten und die Ansprache potentieller Mitstreiter erfolgten in den meisten Fällen im Militär selbst unter der Deckung dienstlicher Abläufe. Ähnlich wie bei der Gruppenkohäsion waren dabei Faktoren wie 
Freundschaften und gemeinsame Erfahrungen, aber auch politische Überzeugungen (Russophilie, Panslawismus) wesentliche Kriterien für die Anwerbung von Unterstützern. Auch wenn die militärische Sphäre den Verschwörern einen gewissen Schutz ermöglichte, ist gleichzeitig zu betonen, dass die Wahrung der Geheimhaltung durch die kurze Vorbereitungszeit gefährdet wurde, die nur relativ oberflächliche Sondierungsgespräche und eine oftmals wenig strikte Eingrenzung des Auditoriums erlaubte.

\section{Fazit}

Das Wirken des konspirativen militärischen Netzwerks in Bulgarien 1886/1887 gestaltete sich in der Spannung zwischen persönlicher Identität, nationalen Prämissen und politischer Loyalität. Gelegentlich harmonierten diese Faktoren miteinander, nicht selten divergierten sie aber in einem so hohen Maße, dass dadurch militärischer Korpsgeist, Berufsethos und Loyalität zum eigenen Staat und zum Monarchen als oberstem Befehlshaber ausgehebelt wurden. Einerseits fühlten sich Offiziere und Mannschaften dem Bund mit dem russischen »Befreier « verpflichtet - viele hatten ihre militärische Ausbildung in Russland erhalten oder absolvierten die von den Russen gegründete und geleitete Militärschule in Sofia und dienten unter russischen Vorgesetzten. Alte Netzwerke und Kameradschaften existierten noch und hatten an Wirksamkeit nicht verloren. Einige Offiziere waren ehemalige Mitglieder der bulgarischen Untergrundorganisation aus den siebziger Jahren des 19. Jahrhunderts, welche für ein unabhängiges Bulgarien kämpfte. Sie unterhielten auch während ihrer militärischen Laufbahn Beziehungen zu früheren Mitstreitern, die ihrerseits selbst russophilen Kreisen nahe standen. Diese Hintergründe prägten eine Loyalitätsgesinnung gegenüber Russland.

Andere Offiziere wiederum wurden durch ihre Vergangenheit als Untergrundkämpfer gegen die Herrschaft des Osmanischen Reiches geprägt und schlossen sich in den achtziger Jahren des 19. Jahrhunderts dem Bulgarischen Geheimen Revolutionären Zentralkomitee an, das die Wiedervereinigung OstRumeliens und Bulgariens anstrebte - ein Ziel, das mit den Interessen Russlands divergierte. Viele von ihnen sahen in Alexander von Battenberg eine Symbolfigur der Souveränität Bulgariens und bekannten sich nach dem militärischen Erfolg gegen Serbien 1885 stärker zur Krone und Battenbergs Politik.

Nach dem Putsch russophiler Offiziere gegen den Fürsten wurde das junge bulgarische Heer in seiner Loyalität erschüttert und gespalten. Der Putsch und seine Hintergründe verdeutlichen die Komplexität und die Sprengkraft geheimer Netzwerke innerhalb des Militärs. Die trennende Wirkung solcher Gruppierungen entfaltete sich im gespaltenen Identitätskonstrukt, welches das bulgarische Heer in den Jahren unmittelbar nach seiner Entstehung charakterisierte. 